\title{
Design of Fuzzy Multiobjective Linear Program Integrated with Fuzzy Vikor for Facility Location
}

\author{
C. Kavitha ${ }^{1 *}$ and C. Vijayalakshmi ${ }^{2}$ \\ 'Department of Mathematics, Sathyabama University, Chennai; ceekavi@gmail.com \\ 2Department of Mathematics, VIT University, Chennai; vijusesha2002@yahoo.co.in
}

\begin{abstract}
Production level of a company increases mainly based on the demand and needs of that location. Facility location models are used to determine the optimal locations based on operating cost, accessibility of the existing, and future facilities. Selection of Location in supply chain for constructing a firm, is based on quantitative and qualitative factors. It is assessed by linguistic variables under fuzzy environment where uncertainty, vagueness, and subjectivity are handled. This paper deals with the design of Fuzzy multi objective Linear Programming model, integrated with Fuzzy VIKOR method. Fuzzy VIKOR method is used for assessing the qualitative factors and mathematical model such as Fuzzy Mixed Integer Linear programming is used for assessing the quantitative factors. Fuzzy VIKOR focuses on ranking \& selecting from a set of alternatives in the presence of conflicting criteria by comparing the measure of "Closeness" to the "ideal" alternative, and thus to discover a best solution. It provides a rational, systematic process, to discover a best solution. It is used to resolve a fuzzy multi-person, multi-criteria decision-making problem. It also allows decision makers to specify the preferred solution for a given decision problem in real organizational settings. Fuzzy Multi Objective Linear Programming is used to find the optimum solution. A case example is illustrated for location selection of a firm, and the results are validated by integrating Fuzzy Mixed Integer Linear Programming with Fuzzy VIKOR method.
\end{abstract}

Keywords: Location Selection, Fuzzy Vikor Method, Fuzzy Multi Objective Linear Program.

\section{Introduction}

Facility location has an important role to play because the site selection directly relates to customers, suppliers, inventory \& material handling. Cheng $\& \mathrm{Li}^{1}$ also used mathematical programme to build a location choice model for distribution centres. Chen ${ }^{2}$ presented a fuzzy group decision model for the allocation of a distribution centre. Cheng \& $\mathrm{Li}^{1}$ also used mathematical programming to build a location choice model for distribution centres. Nicolau ${ }^{3}$ used regression-analysed method to assess new hotel opening through an even study. Teng ${ }^{4}$ applied multi-criteria decision-making method to deal with the site selection for restaurants. Tzeng $^{5}$ developed the multi-criteria selection for a restaurant location in Taipei. Alecos et al. ${ }^{6}$ developed a new technique, based on fuzzy TOPSIS by incorporating the veto threshold as the ultimate reference point for the decision. Opricovic et al. ${ }^{7}$ developed a model for planning the redistribution of industry and residential population throughout the area affected by an earthquake. Saman et al. ${ }^{8}$ developed a Closed-Loop Supply Chain (CLSC) network that is investigated with multiple plants, collection centres, demand markets, products and a mixed-integer linear programming model which minimizes the total cost. A multi-objective optimisation of single-product for four-echelon supply chain architecture solved using swarm intelligence based Multi-objective Hybrid Particle Swarm Optimization (MOHPSO) algorithm by Latha et al. ${ }^{9}$ LixingYang et al. ${ }^{10}$

*Author for correspondence: 
developed a distribution centres location problem with how to select distribution centres from the potential set so that the total relevant cost is minimized. Sanjaya Kumar Behera et al. ${ }^{11}$ developed $\alpha$-cut to solve the multi objective linear programming problems. Hannan et al. ${ }^{12}$ deals with a two-echelon supply chain network design problem in deterministic, single-period, multicommodity contexts. Zhu Yuhua et al. ${ }^{13}$ maintain that the characteristics and requirements in the selection of the location with application of a mathematical model were established on the basis of lowest transportation cost using genetic algorithm.

Using the concept of fuzzy set theory and natural language, the site selection criteria can be evaluated in a more convenient way, allowing decision makers to express their ideas freely and adequately. Therefore, fuzzy set theory, and linguistic value concepts are combined for decision making with the tool to deal with complex issues in fuzzy environment.

\section{Optimization Model for Selection of Location to Construct the Firm}

The goal of the model is to select the best location based on the manufacturing plant, warehouses cost, capacity, demand of the product in the market, qualitative factors etc. Mathematical model is constructed to optimise the best location based on the quantitative and qualitative factors.

It is assumed for discrete set of locations, wherein warehouses and plants are located with different capacity level, with multiple products having different demand in a single period. Products are produced with different raw materials. Different Suppliers supply raw materials. Transportation cost of raw material to each supplier to plant and warehouses, transportation cost of product from plant to warehouse, from warehouse to market are known in advance. Also, location sites for plant and warehouses, Inventory cost per product etc are known. To determine the locations, it is necessary to know the Capacity level of plants and warehouses to deliver the products to retailers, flow of products from plants to warehouses, quantity of raw material shipped from each supplier etc. Fuzzy modelling approach is applied due to imprecise nature of product demand in the market, and decision makers' aspiration level with respect to the goals.

\subsection{Indices}

Locations $(l=1,2 \ldots . L)$

Product $(i=1,2 \ldots . I)$

Plant locations $(j=1,2 \ldots . J)$

Warehouse locations $(w=1,2 \ldots . W)$

Markets $(m=1,2 \ldots . M)$

\subsection{Parameters}

$L \tilde{N}_{j l}^{M P} \quad$ Loan available for constructing manufacturing plant $(j)$ at location $(l)$

$L \tilde{N}_{w l}^{W} \quad$ Loan available for constructing ware house $(w)$ at location $(l)$

$M P E_{l} \quad$ Maximum percentage of loan available at location $(l)$

$\tilde{C}_{i l}^{M P L} \quad$ Unit land cost of manufacturing plant ( $j$ ) at location $(l)$

$\tilde{C}_{w l}^{W L} \quad$ Unit land cost of ware house (w) at location $(l)$

$M A R_{j l}^{M P L}$ Maximum area space available for manufacturing plant $(j)$ at location $(l)$

$M A R_{w l}^{W L} \quad$ Maximum area space available for warehouse $(w)$ at location $(l)$

$\tilde{C}_{j l}^{M P V} \quad$ Unit variable cost of manufacturing plant $(j)$ at location $(l)$

$\tilde{C}_{w l}^{W V} \quad$ Unit variable cost of warehouse $(w)$ at location $(l)$

$\tilde{C}_{j l}^{M P C N} \quad$ Unit construction cost of manufacturing plant $(j)$ at location $(l)$

$\tilde{C}_{w l}^{w C N} \quad$ Unit construction cost of warehouse $(w)$ at location $(l)$

$M R_{j l}^{M P C N}$ Maximum construction requirement for manufacturing plant $(j)$ at location $(l)$

$M R_{w l}^{W C N}$ Maximum construction requirement for warehouse $(w)$ at location $(l)$

$\tilde{C}_{i j l}^{M P O} \quad$ Operating cost for product ( $i$ ) in manufacturing plant $(j)$ at location $(l)$

$\tilde{C}_{i w l}^{W O} \quad$ Operating cost for product (i) in ware house $(w)$ at location $(l)$

$P R_{i j l} \quad$ Processing capacity for the product $(i)$ in manufacturing plant $(j)$ at location $(l)$

$T C_{j l}^{M P} \quad$ Total capacity of the manufacturing plant $(j)$ at location (l)

$T C_{w l}^{W} \quad$ Total capacity of the warehouse $(w)$ at location (l) 
$\tilde{C}_{j l}^{M P L B} \quad$ Unit labour cost of manufacturing plant $(j)$ at location $(l)$

$\tilde{C}_{w l}^{W L B} \quad$ Unit labour cost of ware house $(w)$ at location $(l)$

$\tilde{C}_{i j w l}^{T N M P W}$ Unit transportation cost of product $(i)$ from manufacturing plant $(j)$ to warehouse $(w)$ at location $(l)$

$\tilde{C}_{i w l m}^{W T N} \quad$ Unit transportation cost of product $(i)$ from warehouse $(w)$ at location $(l)$ to market $(m)$

$\tilde{C}_{s i l}^{M P T N}$ Unit transportation cost from supplier $(s)$ to manufacturing plant $(j)$ at location $(l)$

$T C_{s j l} \quad$ Total capacity of the supplier (s) to manufacturing plant $(j)$ at location $(l)$

$\tilde{D}_{i w l m} \quad$ Demand for the product $(i)$ from market $(m)$ at warehouse $(w)$ in location $(l)$

$\tilde{D}_{i j w l} \quad$ Demand for the product $(i)$ from manufacturing plant $(j)$ to warehouse $(w)$ in location $(l)$

$F V_{l} \quad$ Fuzzy VIKOR value for the location $(l)$ based on qualitative factor

\subsection{Decision Variables}

$Y_{l} \quad$ Binary Variable 1 if location $(l)$ is selected or 0 otherwise

$P E_{j l}^{M P} \quad$ Percentage of loan available for constructing manufacturing plant $(j)$ at location $(l)$

$P E_{w l}^{W} \quad$ Percentage of loan available for constructing warehouse $(w)$ at location $(l)$

$A R_{j l}^{M P L} \quad$ Area of manufacturing plant ( $j$ ) at location (l)

$A R_{w l}^{W L} \quad$ Area of warehouse ( $\left.w\right)$ at location (l)

$R_{j l}^{M P C N}$ Construction requirement for manufacturing plant $(j)$ at location $(l)$

$R_{w l}^{W C N} \quad$ Construction requirement for warehouse $(w)$ at location $(l)$

$Q_{i j l}^{M P} \quad$ Quantity of product (i) from manufacturing plant $(j)$ at location $(l)$

$Q_{i w l}^{W} \quad$ Quantity of product (i) from warehouse $(w)$ at location $(l)$

$N_{j l}^{\text {MPLB }} \quad$ Number of labour required for manufacturing plant $(j)$ at location $(l)$

$N_{w l}^{W L B} \quad$ Number of labour required for warehouse $(w)$ at location $(l)$

$M N_{l} \quad$ Maximum number of labour required for location $(l)$

$Q_{s j l}^{M P T N} \quad$ Quantity transported from supplier $(s)$ to manufacturing plant $(j)$ at location $(l)$
$Q_{i j w l}^{T N M P W}$ Quantity of product (i) transported from manufacturing plant $(j)$ to warehouse $(w)$ at location $(l)$

$Q_{i w l m}^{W T N} \quad$ Quantity of product (i) transported from warehouse $(w)$ at location $(l)$ to market $(m)$

\subsection{Objectives of the Formulation}

$$
\begin{gathered}
\text { Min } \mathrm{LAC}+\mathrm{LBC}+\mathrm{VC}+\mathrm{CC}+\mathrm{OP}+\mathrm{TC} \\
\text { Max TLN }+ \text { FV }
\end{gathered}
$$

where LAC $=$ MPLC + WLC

$$
\begin{aligned}
& M P L C=\sum_{l=1}^{L} \tilde{C}_{j l}^{M P L} * A R_{j l}^{M P L}, \quad \forall j \in J \\
& W L C=\sum_{l=1}^{L} \tilde{C}_{w l}^{W L} * A R_{w l}^{W L}, \quad \forall w \in W \\
& \mathrm{LBC}=\mathrm{MPLBC}+\mathrm{WLBC}
\end{aligned}
$$

$$
\begin{aligned}
& M P L B C=\sum_{l=1}^{L} \tilde{C}_{j l}^{M P L B} * N_{j l}^{M P L B}, \quad \forall j \in J \\
& W L B C=\sum_{l=1}^{L} C_{w l}^{W L B} * N_{w l}^{W L B}, \quad \forall w \in W
\end{aligned}
$$

$\mathrm{VC}=\mathrm{MPVC}+\mathrm{WVC}$

$$
\begin{aligned}
& M P V C=\sum_{l=1}^{L} \tilde{C}_{j l}^{M P V} * Y_{l}, \forall j \in J \\
& W V C=\sum_{l=1}^{L} \tilde{C}_{w l}^{W V} * Y_{l}, \forall w \in W
\end{aligned}
$$

$\mathrm{CC}=\mathrm{MPCC}+\mathrm{WCC}$

$$
\begin{aligned}
& M P C C=\sum_{l=1}^{L} \tilde{C}_{j l}^{M P C N} * R_{j l}^{M P C N}, \forall j \in J \\
& W C C=\sum_{l=1}^{L} \tilde{C}_{w l}^{W C N} * R_{w l}^{W C N}, \quad \forall w \in W \\
& \mathrm{OP}=\mathrm{MPOC}+\mathrm{WOC} \\
& M P O C=\sum_{i=1}^{I} \tilde{C}_{i j l}^{M P O} * Q_{i j l}^{M P}, \quad \forall j \in J, \forall l \in L \\
& W O C=\sum_{i=1}^{I} \tilde{C}_{i w l}^{W O} * Q_{i w l}^{W}, \quad \forall w \in W, \forall l \in L
\end{aligned}
$$

$\mathrm{TC}=\mathrm{MPTC}+\mathrm{WTC}$

$$
\begin{aligned}
& M P T C=\sum_{s=1}^{S} \sum_{j=1}^{J} \tilde{C}_{s j l}^{M P T N} * Q_{s j l}^{M P T N}+\sum_{j=1}^{J} \sum_{w=1}^{W} \tilde{C}_{i j w l}^{T N M P W} * Q_{i j w l}^{T N M P W}, \\
& \forall i \in I, \forall l \in L \\
& W T C=\sum_{w=1}^{W} \tilde{C}_{i w l m}^{W T N} * Q_{i w l m}^{W T N}, \quad \forall i \in I, \quad \forall l \in L, \forall m \in M
\end{aligned}
$$




$$
\begin{aligned}
& \mathrm{TLN}=\mathrm{MPL}+\mathrm{WL} \\
& M P L=\sum_{l=1}^{L} L \tilde{N}_{j l}^{M P} P E_{j l}^{M P}, \forall j \in J \\
& W L=\sum_{l=1}^{L} L \tilde{N}_{w l}^{W} P E_{w l}^{W}, \forall w \in W \\
& F V=\sum_{l=1}^{L} F V_{l} * Y_{l}
\end{aligned}
$$

\subsection{Constraints are as Follows}

Loan Constraint:

$$
\sum_{l=1}^{L}\left[P E_{j l}^{M P}+P E_{w l}^{W}\right]=M P E_{l}, \quad \forall j \in J, \quad \forall w \in W
$$

Land Constraint:

$$
\begin{aligned}
& \sum_{l=1}^{L} A R_{j l}^{M P L} \leq M A R_{j l}^{M P L}, \forall j \in J \\
& \sum_{l=1}^{L} A R_{w l}^{W L} \leq M A R_{w l}^{W L}, \quad \forall w \in W
\end{aligned}
$$

\section{Construction Constraint:}

$$
\begin{aligned}
& \sum_{l=1}^{L} R_{j l}^{M P C N} \leq M R_{j l}^{M P C N}, \forall j \in J \\
& \sum_{l=1}^{L} R_{w l}^{W C N} \leq M R_{w l}^{W C N}, \forall w \in W
\end{aligned}
$$

\section{Operating Constraint:}

$$
\begin{gathered}
\sum_{i=1}^{I} P R_{i j l} Q_{i j l}^{M P} \leq T C_{j l}^{M P} Y_{l}, \forall l \in L, \forall j \in J \\
\sum_{l=1}^{L} Q_{i w l}^{W} \leq T C_{w l}^{W} Y_{l}, \forall i \in I, \forall w \in W
\end{gathered}
$$

Labour Constraint:

$$
\sum_{l=1}^{L}\left[N_{j l}^{M P L B}+N_{w l}^{W L B}\right] \leq M N_{l}, \quad \forall j \in J, \quad \forall w \in W
$$

\section{Transportation Constraint}

$$
\begin{gathered}
\sum_{s=1}^{S} Q_{s j l}^{M P T N} \leq T C_{s j l}, \forall j \in J, \forall l \in L \\
\sum_{i=1}^{I} \sum_{l=1}^{L} Q_{i j w l}^{T N M P W} \leq \tilde{D}_{i j w l}, \forall j \in J, \quad \forall w \in W \\
\sum_{i=1}^{I} \sum_{l=1}^{L} Q_{i w l m}^{W T N} \leq \tilde{D}_{i w l m}, \forall w \in W, \quad \forall m \in M
\end{gathered}
$$

$$
\begin{gathered}
\sum_{i=1}^{I} \sum_{l=1}^{l} Q_{i w l m}^{W T N} \leq T C_{w l}^{W}, \forall w \in W, \forall m \in M \\
\sum_{s=1}^{S} Q_{s j l}^{M P T N}-\sum_{i=1}^{I} Q_{i j w l}^{T N M P W} \geq 0, \forall j \in J, \forall s \in S, \forall l \in L, \forall w \in W \\
\sum_{i=1}^{I} Q_{i j w l}^{T N M P W}-\sum_{i=1}^{I} Q_{i w l m}^{W T N} \geq 0, \forall w \in W, \forall j \in J, \forall l \in L \\
\sum_{l=1}^{L} Y_{l} \leq 1
\end{gathered}
$$

Non-negativity Constraint:

$$
Y_{l} \in\{0,1\} \text { for } l=1,2 \ldots \ldots \ldots . L
$$

$$
\begin{aligned}
& P E_{j l}^{M P}, P E_{w l}^{W}, A R_{j l}^{M P L}, A R_{w l}^{W L}, R_{j l}^{M P C N}, R_{w l}^{W C N}, \\
& Q_{i j l}^{M P}, Q_{i w l}^{W}, N_{j l}^{M L B}, N_{w l}^{W L B}, N_{w l}^{W L B}, M N_{l}, Q_{s j l}^{M P T N}, \\
& Q_{i j w l}^{T N M P W}, Q_{i w l m}^{W T N} \geq 0
\end{aligned}
$$

The First Objective function minimizes the investment in operating cost, transportation cost, land cost, labour cost, construction cost, and variable cost. The Second Objective function maximizes the qualitative factors of the location obtained by Fuzzy VIKOR method, Percentage of loan available for constructing manufacturing plant and warehouse corresponding to the location. Constraint (3) ensures that percentage of loan available for both warehouse and plant must be equal to the loan available for the location. Constraint (4) and (5) ensures that maximum area space available for manufacturing plant and warehouse for each location. Constraint (6) and (7) ensures that maximum construction requirement for manufacturing plant, warehouse at location. Processing capacity of the product and the quantity obtained in the manufacturing plant must be less than or equal to the total capacity of the location denotes the constraint (8) and (9). Constraint (10) states that number of labours required for the location. Constraint (11) states the capacity of the supplier. Constraint (12) ensures that manufacturing plant satisfies demand from warehouses. Constraint (13) ensures that warehouses satisfy demand from market. Constraint (14) denotes that quantity of product transported from warehouse to market must be less than or equal to the total capacity of the warehouse in that location. Constraint (15) states that quantity transported from manufacturing plant cannot exceed the amount 
of raw materials received from supplier. Constraint (16) states that quantity transported to market cannot exceed the amount of product from warehouse. Constraint (17) states that location is selected or not. Constraint (18 \& 19) describes the non-negativity constraint and also denotes whether each location is selected or not.

\section{Solution to the Optimization Model for Location Selection}

Factors for the facility location are listed as quantitative and qualitative. Qualitative factors are calculated by Fuzzy VIKOR method, and quantitative factors are based on cost. To find the optimal solution of the location, both the factors are integrated and calculated using Fuzzy MultiObjective Mixed Integer Program.

The Present model is the integration of Fuzzy VIKOR method for qualitative factors with Fuzzy Multi-Objective Mixed Integer Programming Model for quantitative factors. This formulation is to maximize the profit, based on the quantitative and qualitative factors. A comparative analysis is conducted in different location to construct the firm. After preliminary analysis, five locations remain for further evaluation. Those areas would then be subjected to further empirical research by the integrated method to choose the best long-range location.

\section{Phase 1. Fuzzy Vikor Method}

The multi-criteria optimisation and compromise solution called VIKOR is a suitable tool to evaluate each alternative for each criterion function. Fuzzy VIKOR allows decision makers to specify the preferred solution for a given decision problem in real organizational settings. The procedure of fuzzy VIKOR consists of the following steps.

STEP 1: Generate feasible alternatives (denoted in $\mathrm{m}$ ), determine the evaluation criteria (denoted in $\mathrm{k}$ ), and form a group a decision makers (denoted in $\mathrm{n}$ ).

STEP 2: Define linguistic variables \& their corresponding triangular fuzzy number linguistic variables were used to evaluate the importance of the criteria and the ratings of alternatives with respect to various criteria.

STEP 3: Pull the decision makers opinions to get the aggregated fuzzy weight of criteria, and aggregated fuzzy rating of alternatives. If there are ' $n$ ' persons in a decision committee, the importance weight of each criterion and rating of each alternative can be measured by

$$
\begin{gathered}
\tilde{\mathrm{w}}_{\mathrm{j}}=\frac{1}{\mathrm{k}}\left[\tilde{\mathrm{w}}_{\mathrm{j}}^{1} \oplus \tilde{\mathrm{w}}_{\mathrm{j}}^{2} \oplus \ldots \oplus \tilde{\mathrm{w}}_{\mathrm{j}}^{\mathrm{k}}\right] \\
\tilde{x}_{\mathrm{ij}}=\frac{1}{\mathrm{k}}\left[\tilde{x}_{i j}^{1} \oplus \tilde{x}_{i j}^{2} \oplus \ldots \oplus \tilde{x}_{i j}^{k}\right]
\end{gathered}
$$

STEP 4: Construct a fuzzy decision matrix. Fuzzy multicriteria decision making problem can be expressed in matrix format as

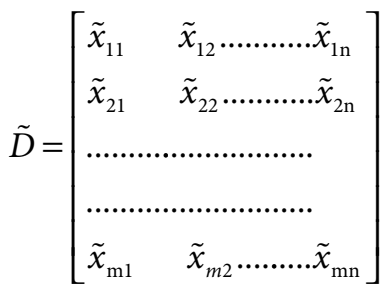

$$
\begin{aligned}
& \mathrm{i}=1,2 \ldots \ldots \mathrm{m} \\
& \mathrm{j}=1,2 \ldots \ldots . \mathrm{n} \\
& \tilde{\mathrm{w}}=\tilde{\mathrm{w}}_{1}, \tilde{\mathrm{w}}_{2} \ldots \ldots \ldots \ldots . . \tilde{\mathrm{w}}_{\mathrm{n}}
\end{aligned}
$$

Where $\tilde{x}_{\mathrm{ij}}$ is the rating of alternatives $\mathrm{A}_{i}$ with respect of $C_{j}, \quad \tilde{w}_{j}$ the importance weight of the $j^{\text {th }}$ criterion holds, $\tilde{x}_{\mathrm{ij}} \& \quad \tilde{w}_{\mathrm{j}}$ is linguistic variable denoted by triangular fuzzy numbers.

STEP 5: Determine the fuzzy best value (FBV, $\left.\tilde{f}_{j}^{*}\right)$ and fuzzy worst value (FWV, $\tilde{\mathrm{f}}_{\mathrm{j}}^{-}$) of all criteria functions.

$$
\begin{aligned}
& \tilde{\mathrm{f}}_{\mathrm{j}}^{*}=\max _{\mathrm{i}} \tilde{x}_{i j} \\
& \tilde{\mathrm{f}}_{\mathrm{j}}^{-}=\min _{\mathrm{i}} \tilde{x}_{i j}
\end{aligned}
$$

STEP 6: Calculate the values $\tilde{s}_{\mathrm{i}}, \tilde{R}_{i}$

$$
\tilde{\mathrm{s}}_{\mathrm{i}}=\sum_{\mathrm{j}=1}^{\mathrm{k}} \tilde{\mathrm{w}}_{\mathrm{j}}\left(\tilde{\mathrm{f}}_{\mathrm{j}}^{*}-\tilde{\mathrm{x}}_{\mathrm{ij}}\right) /\left(\tilde{\mathrm{f}}_{\mathrm{j}}^{*}-\tilde{\mathrm{f}}_{\mathrm{j}}^{-}\right)
$$

where $\tilde{s}_{i}$ refers to the separation measure of $\mathrm{A}_{i}$ from the fuzzy best value. Similarly $\tilde{R}_{i}$ is the separation measure of $A_{i}$ from the fuzzy worst value.

STEP 7: Calculate the values $\tilde{S}^{*}, \tilde{S}^{-}, \tilde{R}^{*}, \tilde{R}^{-} \& \tilde{Q}_{i}$

$$
\begin{gathered}
\tilde{\mathrm{S}}^{*}=\min _{i} \tilde{\mathrm{S}}_{\mathrm{i}}, \tilde{\mathrm{S}}^{-}=\max _{i} \tilde{\mathrm{S}}_{\mathrm{i}} \\
\tilde{\mathrm{R}}^{*}=\min _{i} \tilde{\mathrm{R}}_{\mathrm{i}}, \tilde{\mathrm{R}}^{-}=\max _{i} \tilde{\mathrm{R}}_{\mathrm{i}} \\
\tilde{\mathrm{Q}}_{\mathrm{i}}=\mathrm{v}\left(\tilde{\mathrm{S}}_{\mathrm{i}}-\tilde{\mathrm{S}}^{*}\right) /\left(\tilde{\mathrm{S}}^{-}-\tilde{\mathrm{S}}^{*}\right)+(1-\mathrm{V})\left(\tilde{\mathrm{R}}_{\mathrm{i}}-\tilde{\mathrm{R}}^{*}\right) /\left(\tilde{\mathrm{R}}^{-}-\tilde{\mathrm{R}}^{*}\right)
\end{gathered}
$$


Here $\tilde{S}^{*}$ is the minimum value of $\tilde{S}_{i}$ which is the maximum majority rule (or) maximum group utility, and $\tilde{R}^{*}$ is the minimum value of $\tilde{R}_{i}$ which is the minimum individual regret of the opponent. Thus, the index $\tilde{Q}_{i}$ is obtained and is based on the consideration of both the group utility, and individual regret of the opponent.

In addition, $\mathrm{V}$ here means the weight of the strategy of the maximum group utility. When $\mathrm{V}>0.5$, the decision tends towards the maximum majority rule, and if $\mathrm{V}=0.5$, the decision tends towards the individual regret of the opponent usually $\mathrm{V}=0.5$.

STEP 8: Defuzzify triangular fuzzy number $\tilde{Q}_{i}$ and rank the alternative by the index $\tilde{Q}_{i}$.

The process, converting a fuzzy number into a crisp value is called defuzzify. Various defuzzification strategies were suggested in the paper; Chen's (1985) ranking method is applied. The Chen's ranking method (1985) is carried out by computing total utility (or) ordering value $\mathrm{U}_{\mathrm{T}}(\mathrm{x})$ to rank the alternatives. The concept is based on maximizing and minimizing set.

$$
\mathrm{U}_{\mathrm{T}}\left(\mathrm{F}_{\mathrm{i}}\right)=\left[\mathrm{U}_{\mathrm{R}}\left(\mathrm{F}_{\mathrm{i}}\right)+1-\mathrm{U}_{\mathrm{L}}\left(\mathrm{F}_{\mathrm{i}}\right)\right] / 2
$$

The index $Q_{i}$ implies the separation measure of $\mathrm{Ai}$ from the best alternative. (ie) the smaller the value, the better alternative.

STEP 9: Determine a compromise solution.

Assume that the two conditions given below are acceptable. Then, by using the index, $Q_{i}$ determines a compromise solution (a') as a single optimal solution.

(Condition 1) Acceptable advantage;

$$
\mathrm{Q}\left(\mathrm{a}^{\prime \prime}\right)-\mathrm{Q}\left(\mathrm{a}^{\prime}\right) \geq \mathrm{DQ}, \mathrm{DQ}=\frac{1}{\mathrm{~m}-1}[\mathrm{DQ}=0.25 \text { if } \mathrm{m} \leq 4]
$$

(Condition 2) Accepted stability in decision making

$$
\mathrm{Q}\left(\mathrm{a}^{\prime}\right) \text { must in S (a') or/and R (a') }
$$

If condition 1 is not satisfied, $Q\left(a^{m}\right)-Q\left(a^{\prime}\right)<D Q$, $a^{\text {' }}$ $\mathrm{a}^{\prime \prime} \ldots \mathrm{a}^{(\mathrm{m})}$ are compromise solutions in the same. If condition 2 is not satisfied, a' \& a" are compromise solution in the same.

STEP 10: Determine the best alternative. The best alternative is $Q\left(a^{\prime}\right)$, which is one with the minimum of $Q_{i}$.

\section{Phase 2. Fuzzy Multi Objective Linear Programming}

Fuzzy multi objective linear programming is applied to the real life problems, which simultaneously solve the decision maker's vague goals and optimise the goals using membership functions. It also used to solve if the input data are uncertain in nature, and the decision makers contain some aspiration level for the goals and constraints.

A multi objective linear programming model can be stated as

Find $X=\left(x_{1}, x_{2}, \ldots \ldots \ldots \ldots \ldots x_{n}\right)^{T}$. So as to maximize or mini-

mize

$$
\begin{aligned}
& Z_{k}(X)=\sum_{j=1}^{n} C_{j}^{k} x_{j}, k=1,2 \ldots \ldots \ldots \ldots \ldots . . K \\
& \quad \text { Such that } \sum_{j=1}^{n} a_{i j} x_{j}(\leq,=, \geq) \quad b_{i}, \quad i=1,2 \ldots \ldots \ldots \ldots . . m \\
& x_{j} \geq 0, \quad j=1,2 \ldots \ldots \ldots \ldots . n
\end{aligned}
$$

Here, the objective functions are conflicting in nature. It is assumed that the problem is feasible; and there exists an optimal compromise solution. Therefore, we apply fuzzy programming approach to find an optimal compromise solution. In Fuzzy programming approach, aspiration level of the goals is determined by decision makers. It needs maximum and minimum limits for each goal. The upper $\left(\mathrm{U}_{\mathrm{k}}\right)$ and lower $\left(\mathrm{L}_{\mathrm{k}}\right)$ values for each goal are calculated using payoff table. Thus, feasibility of each fuzzy goal is determined.

Here, the $\mathrm{mth}$ objective function is denoted as $\mathrm{Z}_{\mathrm{m}}(\mathrm{X})$, and the corresponding optimal solution for the $m$ th single objective is $\mathrm{X}_{(\mathrm{m})}$. For each objective, a payoff matrix with lower bound and upper bound is formulated (i.e) $\mathrm{U}_{\mathrm{m}}=$ $\max \left[\mathrm{Z}_{1 \mathrm{~m}}, \mathrm{Z}_{2 \mathrm{~m}}, \ldots . . \mathrm{Z}_{\mathrm{Mm}}\right]$ and $\mathrm{L}_{\mathrm{m}}=\min \left[\mathrm{Z}_{1 \mathrm{~m}}, \mathrm{Z}_{2 \mathrm{~m}}, \ldots . . \mathrm{Z}_{\mathrm{Mm}}\right]$ are presented in Table 1.

Membership functions are developed for each goal. Using Zimmermann max, min operator approach, fuzzy feasible optimal solution for the goals or fuzzy constraints is obtained by intersection of membership

\begin{tabular}{|c|c|c|c|c|}
\hline & $\mathrm{Z}_{1}(\mathrm{X})$ & $\mathrm{Z}_{2}(\mathrm{X})$ & ....................... & $\mathrm{Z}_{\mathrm{M}}(\mathrm{X})$ \\
\hline $\mathrm{X}^{(1)}$ & $Z_{11}$ & $Z_{12}$ & & $Z_{1 M}$ \\
\hline$X^{(2)}$ & $Z_{21}$ & $Z_{22}$ & & $Z_{2 M}$ \\
\hline$\cdots$ & $\cdots$ & 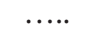 & …........... & $\cdots \cdots$ \\
\hline $\mathrm{X}^{(\mathrm{M})}$ & $\mathrm{Z}_{\mathrm{M} 1}$ & $\mathrm{Z}_{\mathrm{M} 2}$ & & $\mathrm{Z}_{\mathrm{MM}}$ \\
\hline
\end{tabular}
functions. The solution set is characterized by the membership function as $\mu_{D}(x)=\left\{\left\{\cap \mu_{z_{k}}(x)\right\} \cap\left\{\cap \mu_{g_{r}}(x)\right\}\right\}$

The optimal solution is given by

$$
\mu_{D}\left(x^{*}\right)=\max _{x \in X_{d}} \mu_{D}(x)=\max _{x \in X_{d}} \min \left[\min _{k=1,2 \ldots K} \mu_{z_{k}}(x), \min _{r=1,2 . \ldots h} \mu_{g_{r}}(x)\right] .
$$

Table 1. Payoff Matrix 
In order to find optimal solution, formulate as a crisp model by introducing an augmented variable $\lambda$. Then, it can be transformed into following conventional linear programming problem as

Maximize $\lambda$

$$
\begin{aligned}
& \lambda \leq \mu_{z_{k}}(x), k=1,2 \ldots . . K \text { [Objective functions] } \\
& \lambda \leq \mu_{g_{r}}(x), r=1,2 \ldots \ldots \ldots . . . h \text { [Fuzzy constraints] } \\
& g_{p}(x) \leq b_{p}, p=h+1, \ldots \ldots \ldots . . . m \text { [Deterministic constraints] } \\
& x_{j} \geq 0, \quad j=1,2 \ldots \ldots \ldots \ldots . . . . \\
& \lambda \in[0,1]
\end{aligned}
$$

Fuzzy Multi objective linear Programming approach can be summarized in the following steps:

STEP 1: Formulate the mathematical model of crisp linear program.

STEP 2: Obtain the solution for each objective function and construct the payoff table. If the decision maker is satisfied by the preferred solution, stop the process. Otherwise go to Step 3.

STEP 3: Membership functions for each objective are constructed using the lower and upper bound.

STEP 4: Formulate the fuzzy linear program.

STEP 5: Obtain a compromise solution. If the solution is acceptable by the decision maker, stop the process, otherwise, modify the lower, upper bound and repeat the process.

\section{Numerical Calculations and Graphical Representations for Location Selection}

In order to illustrate the model, a hypothetical case study of 5 locations, 5 manufacturing plants, 5 warehouses, 3 suppliers, 2 products, 2 markets has been considered. Here, imprecision in the decision makers aspiration level for goals and product demand in the market are considered simultaneously and fuzzy approach is used. The model is analysed with two conflicting objectives as maximizing the qualitative factors, loan for the location, and minimizing the total cost for the location. This model depends on the value judgement of the decision maker. These demands will change every year, depending upon the product demand and cost.

Qualitative factors are evaluated using fuzzy methods. Committees of three decision makers D1, D2 and D3 have been formed to select the most suitable location. Criteria are (C1) Export \& Import Regulations, (C2) Industrial
Relations, (C3) Quality of life, (C4) Technical skills, and (C5) Safety. These criteria's are evaluated by Fuzzy VIKOR method to solve Fuzzy multi-criteria decision-making process.

\section{Fuzzy VIKOR Method}

STEP 1: Form a group of decision-makers; determine the evaluation criteria and feasible alternatives. In this case, there are three decision makers, five evaluation criteria, and five locations.

STEP 2: Identify the appropriate linguistic variables for the importance weight of criteria, and the rating of alternatives with regard to each criterion, and alternative as shown in Table 2 and Table 3.

Table 2. Linguistic variables for the weight of criteria

\begin{tabular}{ll}
\hline Linguistic Variables & Fuzzy Numbers \\
\hline Very Low $(\mathrm{VL})$ & $(0.00,0.00,0.25)$ \\
Low $(\mathrm{L})$ & $(0.00,0.25,0.50)$ \\
Medium(M) & $(0.25,0.50,0.75)$ \\
$\operatorname{High}(\mathrm{H})$ & $(0.50,0.75,1.00)$ \\
Very High $(\mathrm{VH})$ & $(0.75,1.00,1.00)$ \\
\hline
\end{tabular}

Table 3. Linguistic variables for the ratings of alternative

\begin{tabular}{ll}
\hline Linguistic Variables & Fuzzy Numbers \\
\hline Worst(W) & $(0.0,0.0,2.5)$ \\
Poor(P) & $(0.0,2.5,5.0)$ \\
Fair(F) & $(2.5,5.0,7.5)$ \\
Good(G) & $(5.0,7.5,10)$ \\
$\operatorname{Best}(\mathrm{B})$ & $(7.5,10,10)$ \\
\hline
\end{tabular}

STEP 3: Pull the decision maker's opinions. The importance weight of the criteria and the rating of locations on five criteria are expressed in Table $4 \& 5$.

Table 4. The Importance weight of the criteria

\begin{tabular}{cccc}
\hline & D1 & D2 & D3 \\
\hline C1 & VH & VH & VH \\
C2 & H & VH & H \\
C3 & H & M & M \\
C4 & H & H & H \\
C5 & VH & H & VH \\
\hline
\end{tabular}


Table 5. Rating of the locations by the decision makers under the various criteria

\begin{tabular}{lccccc}
\hline Criteria & L1 & L2 & L3 & L4 & L5 \\
\hline C1 & (B, G, G) & (G, F, P) & (F, F, W $)$ & (F, F, P) & (G, G, B) \\
C2 & (B, G, B) & (P, W, P) & (P, P, F $)$ & (F, G, F) & (F, F, P) \\
C3 & (B, B, B) & (F, P, F) & (G, G, B) & (G, B, B) & (P, P, W) \\
C4 & (B, F, G) & (F, G, G) & (P, P, W $)$ & (P, P, F) & (F, P, F) \\
C5 & (P, P, P) & (G, B, G) & (F, F, G) & (F, F, F) & (F, G, B) \\
\hline
\end{tabular}

STEP 4: Aggregate fuzzy weight of criteria \& fuzzy ratings of location to construction the aggregated fuzzy decision matrix (addressed in Table 6 \& 7).

Table 6. Aggregated fuzzy weight of criteria

\begin{tabular}{lc}
\hline Criteria & Weight \\
\hline C1 & $(0.75,1.00,1.00)$ \\
C2 & $(0.58,0.83,1.00)$ \\
C3 & $(0.33,0.58,0.83)$ \\
C4 & $(0.50,0.75,1.00)$ \\
C5 & $(0.67,0.92,1.00)$ \\
\hline
\end{tabular}

Table 7. Aggregated fuzzy decision matrix

\begin{tabular}{cccccc}
\hline & L1 & L2 & L3 & L4 & L5 \\
\hline \multirow{4}{*}{ C1 } & $(5.83$, & $(2.5,5.0$, & $(1.67$, & $(1.67$, & $(5.83$, \\
& 8.33, & $7.5)$ & 3.33, & 4.17, & 8.33, \\
& $10)$ & & $5.83)$ & $6.67)$ & $10)$ \\
& $(6.67$, & $(0.0,1.67$, & $(0.83$, & $(3.33$, & $(1.67$, \\
C2 & 9.17, & $4.17)$ & 3.33, & 5.83, & 4.17, \\
& $10)$ & & $5.83)$ & $8.33)$ & $6.67)$ \\
& & $(1.67$, & $(5.83$, & $(6.67$, & $(0.0$, \\
C3 & $(7.5,10$, & 4.17, & $8.33,10)$ & $9.17,10)$ & 1.67, \\
& $10)$ & $6.67)$ & & & $4.17)$ \\
& & $(4.17$, & $(0.0,1.67$, & $(0.83$, & $(1.67$, \\
C4 & $(5,7.5$, & 6.67, & $4.17)$ & 5.83, & 4.17, \\
& $9.17)$ & $9.17)$ & & & \\
& & & $(3.63$, & $(2.5,5.0$, & $(5,7.5$, \\
C5 & $(0.0,2.5$, & $(5.83$, & 5.83, & $7.5)$ & $9.17)$ \\
& $5.0)$ & $8.33,10)$ & $8.33)$ & & \\
\hline
\end{tabular}

STEP 5: Determine the fuzzy best value (FBV, $\left.\tilde{\mathrm{f}}_{j^{*}}\right)$ \& fuzzy worst value (FWV, $\tilde{\mathrm{f}}_{j-}$ ) as in Table 8.
Table 8. Fuzzy Best value (FBV) \& fuzzy worst value (FWV)

\begin{tabular}{lcc}
\hline Criteria & $\tilde{\mathrm{f}}_{j^{*}}$ & $\tilde{\mathrm{f}}_{j-}$ \\
\hline C1 & $(5.83,8.33,10)$ & $(1.67,3.33,5.83)$ \\
C2 & $(6.67,9.17,10)$ & $(0.0,1.67,4.17)$ \\
C3 & $(7.5,10,10)$ & $(0.0,1.67,4.17)$ \\
C4 & $(5,7.5,9.17)$ & $(0.0,1.67,4.17)$ \\
C5 & $(5.83,8.33,10)$ & $(0.0,2.5,5.0)$ \\
\hline
\end{tabular}

STEP 6: Values $\tilde{S}_{i} \& \tilde{R}_{i}$ are computed respectively as shown in Table 9.

Table 9. Index $\tilde{\mathrm{S}}_{\mathrm{i}} \& \tilde{\mathrm{R}}_{\mathrm{i}}$

\begin{tabular}{llllll}
\hline & L1 & L2 & L3 & L4 & L5 \\
\hline$\tilde{S}_{\mathrm{i}}$ & $(0.67$, & $(1.519$, & $(2.119$, & $(1.877$, & $(1.193$, \\
& $0.92,1)$ & 2.008, & 2.9071, & 2.321, & 1.693, \\
& & $2.074)$ & $3.049)$ & $2.253)$ & $2.067)$ \\
$\tilde{\mathrm{R}}_{\mathrm{i}}$ & $(0.67$, & $(0.6004$, & $(0.75$, & $(0.75$, & $(0.435$, \\
& $0.92,1)$ & 0.83, & 1.0, & 0.832, & $0.58,0.83)$ \\
& & $1.0)$ & $1.0)$ & $0.798)$ & \\
\hline
\end{tabular}

STEP 7: $\tilde{\mathrm{S}}_{*}, \tilde{\mathrm{S}}_{-}, \tilde{\mathrm{R}}_{*}, \tilde{\mathrm{R}}_{-}$can be seen in Table 10.

Table 10. $\quad \tilde{\mathrm{S}}_{*}, \tilde{\mathrm{S}}_{-}, \tilde{\mathrm{R}}_{*}, \tilde{\mathrm{R}}_{-}$

\begin{tabular}{cc}
\hline$\tilde{\mathrm{S}}_{*}$ & $(0.67,0.92,1.0)$ \\
$\tilde{\mathrm{S}}_{-}$ & $(2.119,2.907,3.049)$ \\
$\tilde{\mathrm{R}}_{*}$ & $(0.435,0.58,0.798)$ \\
$\tilde{\mathrm{R}}_{-}$ & $(0.75,1.0,1.0)$ \\
\hline
\end{tabular}

STEP 8: Calculate $\tilde{Q}_{i}$ for each location \& the result is shown in Table 11.

Table 11. Index $\tilde{Q}_{i}$ and Rank for Locations

\begin{tabular}{lccc}
\hline Location & $\tilde{Q}_{i}$ & $\mathrm{UT}\left(\tilde{Q}_{i}\right)=\mathrm{FVj}$ & Rank \\
\hline L1 & $(0.373,0.405,0.50)$ & 0.282 & 2 \\
L2 & $(0.555,0.572,0.763)$ & 0.522 & 3 \\
L3 & $(1.0,1.0,1.0)$ & 1.000 & 5 \\
L4 & $(0.916,0.653,0.306)$ & 0.557 & 4 \\
L5 & $(0.181,0.194,0.339)$ & 0.090 & 1 \\
\hline
\end{tabular}

STEP 9: Determine a compromise solution (a') by the index $\mathrm{Q}$ in double condition. 
(Condition 1) Acceptable advantage: $\mathrm{Q}\left(\mathrm{a}^{\prime}\right)-\mathrm{Q}\left(\mathrm{a}^{\prime}\right)=$ $0.192>0.25$.

(Condition 2) Acceptable stability in decision making in Table 12.

Table 12. Acceptable stability in decision making

\begin{tabular}{llllll}
\hline Rank by $\mathrm{Q}_{\mathrm{i}}$ & L5 & L1 & L2 & L4 & L3 \\
${\text { Rank by } \mathrm{S}_{\mathrm{i}}}_{\text {Rank by } \mathrm{R}_{\mathrm{i}}}$ & L1 & L5 & L2 & L4 & L3 \\
\hline
\end{tabular}

STEP 10: The best alternative is determined. L5 is the best alternative owing to its closeness, and is the best location.

Membership functions of fuzzy objectives using lower and upper bound as follows

$$
\begin{gathered}
\mu_{\text {TCOST }}= \begin{cases}0 & \text { if } \quad \text { TCOST }>3,239,000 \\
\frac{3,239,000-\mathrm{TCOST}}{3,239,000-2,710,555} & \text { if } \quad 2,710,555<\mathrm{TCOST} \leq 3,239,000 \\
1 & \text { if } \quad \text { TCOST } \leq 2,710,555\end{cases} \\
\mu_{\text {LOAN }}=\left\{\begin{array}{lll}
1 & \text { if } \quad \text { LOAN }>75,835 \\
\frac{\text { LOAN-65,000 }}{75,835-65,000} & \text { if } & 65,000<\mathrm{LOAN} \leq 75,835 \\
0 & \text { if } & \text { LOAN } \leq 65,000
\end{array}\right. \\
\mu_{\text {DEMAND }}= \begin{cases}1 & \text { if } \quad \text { DEMAND } \leq 10000 \\
1-\frac{\text { DEMAND-10,000 }}{1000} & \text { if } \quad 10,000 \leq D E M A N D \leq 11,000 \\
0 & \text { if } \quad \text { DEMAND } \geq 11,000\end{cases}
\end{gathered}
$$

Table 13. The Payoff Table

\begin{tabular}{lcc}
\hline & TCOST & LOAN \\
\hline TCOST & $2,710,555$ & 65,000 \\
LOAN & $3,239,000$ & 75,835 \\
\hline
\end{tabular}

Table 14. Lower and Upper bound for the Objectives

\begin{tabular}{lcc}
\hline Objectives & Lower bound & Upper bound \\
\hline TCOST & $2,710,555$ & $3,239,000$ \\
LOAN & 65,000 & 75,835 \\
\hline
\end{tabular}

Table 15. Compromise Solution

\begin{tabular}{|c|c|c|c|c|}
\hline$\lambda$ & TCOST & LOAN & $\mu_{T C O S T}$ & $\mu_{\text {LOAN }}$ \\
\hline 0.5518 & 2948351 & 71131 & 0.5518 & 0.5518 \\
\hline
\end{tabular}

The lower and upper bound of the objectives are determined as payoff table in Table 13. The solution space which corresponds the maximum and minimum values are determined as lower and upper bounds respectively, and presented in Table 14. Compromise solution are presented in Table 15. The solution, obtained by the mathematical model will help the decision maker to develop management attitude in changing environment. Figure 1 represents the ranking of location in Fuzzy VIKOR method, and Figure 2 represents the compromise solution of the objectives by Fuzzy Multi objective Linear Program.

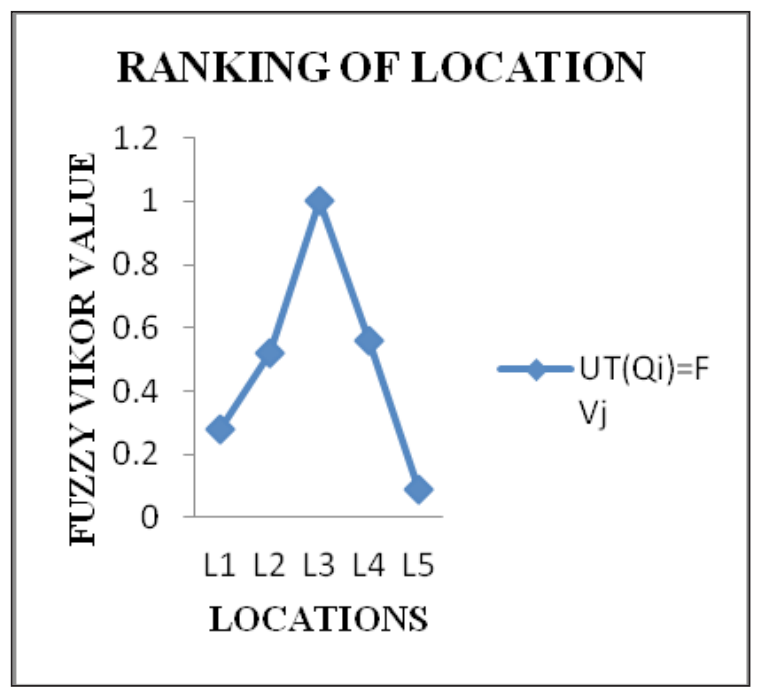

Figure 1. Ranking the location based on group utility and individual regret of the opponent by Fuzzy Vikor Method.

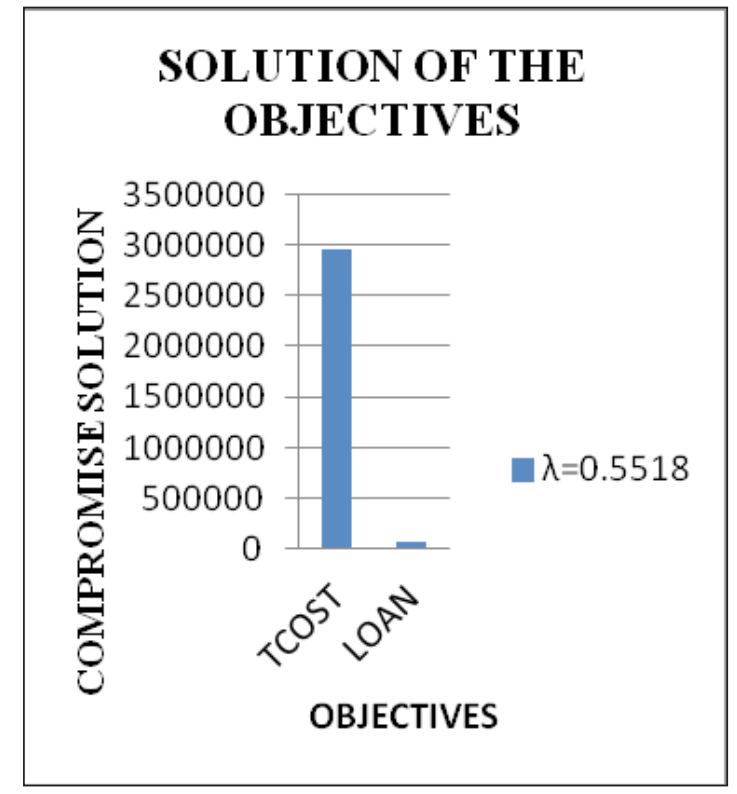

Figure 2. Compromise solution of the objectives. 


\section{Conclusion}

Location Selection for constructing a firm is a long-term plan in an organization. Key role in strategic planning of an organization is location selection for constructing a plant because of cost involved, and its impact on other managerial decision. Proposed methodology is a combination of both quantitative and qualitative factors in dealing with uncertainty real world optimisation problems. Fuzzy VIKOR is supportive in dealing with situations that are, too, complicated, and chosen location can be accepted due to its maximum group utility of the majority as well as the minimum individual's regret of opponent. A fuzzy multi-objective linear programming model is developed for selecting the best location. The model represents the decision maker's aspiration level in goals and uncertainty values in the constraints, which is impossible by conventional method.

\section{References}

1. Cheng EWL, Li H. Exploring quantitative methods for project location selection. Build Environ. 2004; 39 (12): 1467-1476.

2. Chen CT. A fuzzy group decision model of location selection for distribution center. Journal of Management and Systems. 1999; 6 (4), 459-480.

3. Nicolau JL. Assessing new hotel opening through can even study. Tourism Manage. 2002; 23:47-54.

4. Teng MH. Application of multi-criteria decision making for site selection of restaurants: case study of Pao-San restaurant. Journal of Living Sciences. 2000; 6:47-62.
5. Tzeng GH, Teng MH, Chen JJ, Opricovic S. Multicriteria selection for a restaurant location in Taipei. International Journal of Hospitality Management. 2002; 21:171-187.

6. Kelemenis AM and Askounis DTh. An extension of fuzzy TOPSIS for personnel selection. Proceedings of the 2009 IEEE International Conference on Systems. Man \& Cybernetics. San Antonio, TX USA; 2009.

7. Opricovic S and Tzeng GH. Multicriteria planning of post-earthquake sustainable reconstruction. Computer Aided Civil \& Infrastructure Engineering. 2002; 17(3): 211-220.

8. Amin SH, Zhang G. A multi-objective facility location model for closed-loop supply chain network under uncertain demand and return. Appl Math Model. 2013; 37(2013):4165-4176.

9. Shankar BL, Basavarajappa S, Jason $\mathrm{CH}$, Chen C., Kadadevaramath RS, Location and allocation decisions for multi-echelon supply chain network - a multi-objective evolutionary approach. Expert Syst Appl. 2013; 40(2013): 551-562.

10. LixingYang, Xiaoyu J, Gao Z, Li K. Logistics distribution centers location problem and algorithm under fuzzy environment. J Comput Appl Math. 2013; 208(2007): 303-315.

11. Behera SK, Nayak JR. Solution of multi-objective mathematical programming problems in fuzzy approach, Int J Comput Sci Eng. 2011 Dec; 3(12).

12. Sadjady $\mathrm{H}$ and Davoudpour $\mathrm{H}$. Two-echelon, multicommodity supply chain network design with mode selection, lead-times and inventory costs. Comput Oper Res. 2012; 39(2012):1345-1354.

13. Yuhua Z, Tong Z. Optimization of ocations of Grain logistics centers based on improved Genetic; 2009. 